\title{
Design of Speed Regulation Remote Monitoring System of Belt Conveyor
}

\author{
Wang Ning \\ School of Logistics Engineering \\ Wuhan University of Technology \\ Wuhan, China \\ E-mail: wangning872920@163.com
}

\author{
Hu Juan \\ School of Logistics Engineering \\ Wuhan University of Technology \\ Wuhan, China \\ E-mail: 591436686@qq.com
}

\begin{abstract}
Intuitively display system operation parameters are the basis of the implementation for belt conveyor speed regulation and energy-saving control. This paper presents a speed regulation remote monitoring system of belt conveyors based on PLC and king view technology to meet the requirements of visibility, real-time and accurately for belt conveyor speed regulation remote monitoring. This system consists of a hypogyny machine system which considers hardware module as a unit and an upper machine system considering software entities as a unit. Between the upper machine and the lower machine, this paper uses PPI (Point to - Point) to monitor data remote communication. First, design the structure of speed regulation remote monitoring system of belt conveyor, then design control process of the system, the communications between king view and Siemens S7-200, and king view interface. This system will help to research belt conveyor remote intelligent control of motor speed, then promote energy conservation and emissions reduction about the ports and the enterprises, and realize downsizing efficiency.
\end{abstract}

Keywords- Belt-speed Control; Monitor and Control System; King View; Frequency Conversion Technology; Tape Speed Control

\section{INTRODUCTION}

China is a world ports country, by the end of 2011, China already has 31,968 production berths, and port cargo throughput and container throughput ranked first in the world for nine consecutive years[1]. At the same time, the port original extensive expansion mode has made energy consumption and environmental problem increasingly prominent. The society has more voices about energy saving and resource -saving for the port industry and environment-friendly construction of the port industry in the port. Bulk material handling is the biggest factor which affects the energy consumption of ports.

As a port bulk continuous conveying equipment, belt conveyor system are usually configured according to the maximum traffic, when the belt traffic volume changed, constant speed operation mode will make the serious waste of the system energy. In recent years, with the development of frequency conversion technology, Adjustable belt speed runs great potential for energy saving control technology based on real-time load conditions[2].

Inverter is Electrical control equipment that using frequency conversion technology and microelectronic technology to control the ac motor by changing the way of power frequency motor work. IGBT inside the inverter by breaking to adjust the output voltage and frequency power supply, according to the actual needs of the motor to provide power supply voltage it needs, thus achieving the purpose of energy, speed, in addition, the protection of the inverter and there are many functions, such as over-current, over-voltage protection, overload protection, etc. With the continuous improvement of industrial automation, the inverter also got very extensive application.

In the frequency control, often using voltage source inverter, its working principle is: the voltage and frequency of the three phase alternating current fixed after the uncontrolled rectifier bridge into pulsating direct current, pulsating direct current and then filtered by reverse variable convert DC frequency-tunable, adjustable voltage equivalent AC output to the asynchronous motor. Since the power supply frequency is proportional to motor speed by changing the frequency of the power output, adjust the motor speed, to achieve smooth and stepless speed regulation.

The basic principle of frequency control technology is based on the proportional relationship between the motor speed and the power supply input frequency:

$$
\mathrm{n}=60 \mathrm{f}(1-\mathrm{s}) / \mathrm{p}
$$

$$
\begin{aligned}
& \mathrm{n} \text {-rotational speed } \\
& \mathrm{f} \text { - the input frequency } \\
& \mathrm{s} \text { - motor slip } \\
& \mathrm{p} \text {-motor pole pairs }
\end{aligned}
$$

When the frequency $\mathrm{f}$ is continuously adjustable (usually for a set number of $\mathrm{P}$ ), the motor synchronous speed are continuously adjustable.

For the induction motor rotor speed is always slightly lower than the synchronous speed, therefore, when the synchronous speed continuously adjustable, asynchronous motor rotor speed is also continuously adjustable. The inverter is by changing the $\mathrm{f}$ (frequency current) to make the motor drive.

At present, there are two ways about monitoring belt conveyor system: unit monitoring and centralized monitoring. However, no matter which kinds of means used, monitoring information are not intuitive display belt conveyor operation parameters, unable to meet the need of requirements of remote monitoring, real-time visibility speed regulation and energy saving[3]. Taking into account the above-mentioned problems and the high cost 
about bulk material handling test in the actual bulk handling process, the difficulty is big, the author has developed a set of belt conveyor speed energy-saving remote monitoring test system. The system is based on local / remote switch control mode to build a remote control conveyor system network, Using programmable logic controller (PLC) to complete a remote control cabinet hardware design, and using King view to design the belt conveyor remote control interface, which achieved the center to control remote frequency control conveyor belt speed . System will be good for belt conveyor speed regulation and energy saving control strategy research[4].

\section{MONITORING SYSTEM HARDWARE ARCHITECTURE}

Belt conveyor automatic energy-saving operation, the key to its implementation is by controlling the speed of the conveyor, to implement the conveyor soft start, stop, power balance and energy-saving operation [5].

In this paper, based on PLC, the frequency control of motor speed technology for energy-saving control of the belt conveyor is presented. This system consists of PLC programmable controller, inverter, tape speed sensors and motor current sensor and other components. The system consists of can be roughly divided into the following three units[6]:

- The detection unit. The motor current signal from the current sensor obtained in the motor; tape speed sensors are responsible for collecting the tape speed signal, and then converted into a voltage signal via $\mathrm{f} / \mathrm{v}$, all signals are transmitted to the PLC of the A/D module.

- The control unit. When the PLC receives the detection signal, which will be judged decisions, at the same PLC will complete conveyor soft start, the power balance and energy-saving speed and other functions.

- The execution units. When the inverter receives the frequency control signals from the PLC, will be in accordance with the prescribed the corresponding frequency signal output voltage of the load on the motor, so as to realize the frequency control of motor speed of the motor, thus can complete the functions of belt conveyor.

Remote monitoring system of belt conveyor speed regulation and energy saving structure are shown in Fig. 1.

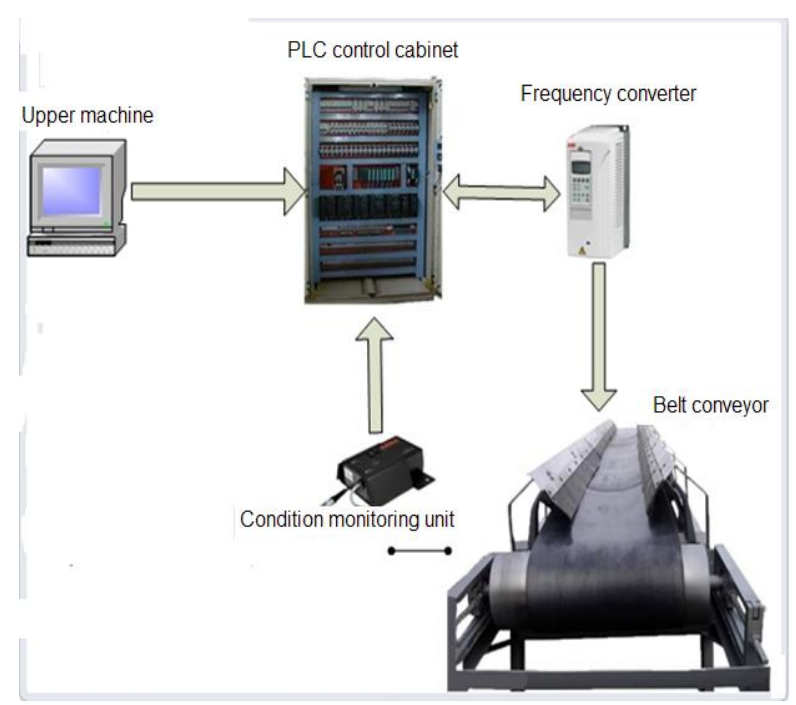

Figure 1. system basic structure

\section{A. Control design of the belt conveyor}

Belt conveyor control circuit diagram of the system is shown in Fig. 2, .

Belt of A and B, respectively, started by the motor M2 and M1, motor M1, M2, respectively are controlled by the contactor KM1, KM2, KM3, KM4. KM1, KM2 control belt A positive \&negative, respectively (KM1 reversal, KM2 is turn), KM3, KM4 respectively control belt B positive \&negative (KM3 reversal, KM4 is turn). SB11 is the reverse start button of belt A; SB12 is the positive start button of belt A; SB13 is the stop button of belt A.SB21 is the start button for the reverse of the belt B; SB22 is the belt $\mathrm{B}$ of the forward start button.

Two electric motors are made short circuit and fuse and the thermal relay overload protection, two in any one overload failure, another electric motor will cease to function.

\section{B. Connection design of upper machine and lower machine}

The control system can be a pair of belt integrated control system. This system has many advantages: parameters set flexible, easy to control, maintainability, a variety of control portfolio, with a belt comprehensive protection and monitoring PC screen and so on, which is a belt conveyor system overall control program of bulk materials.

The system displays the site environment and the quality of materials, belt speed and other parameters through the PC and the control center display, and can be able to power the belt frequency control, to achieve the desired constant speed, and then achieving energy savings.

This system chooses the ABB inverter ACS510-01, this kind of frequency converter has a general software tools with fieldbus general customers and process interface, specification design, debugging and maintenance. It is built RS485 interface, connecting other devices with Modbus protocol, within easy parameter setting, you can use the Modbus RTU protocol. Hardware, 28-32 terminals for RS485 communication of frequency converter, using the shielded twisted-pair cable connection, thus can realize the system communication. 
The system uses a combination of hardware and King view software, through a machine up and collaborative working mode to realize remote belt conveyor speed control, including the hardware module as a unit of a machine system and software entity as the unit of the upper machine system[7]. The machine system with Siemens PLC (Programming Logical Controller) and intelligent control equipment such as $\mathrm{ABB}$ inverter as the core, completed the control of the motor controlled object.
Upper machine system uses king view software as the platform, for communication up and down the monitor bit machines connected via field bus technology, and then collect data through man-machine interface in front of the operator to complete the real-time monitoring of the belt conveyor.

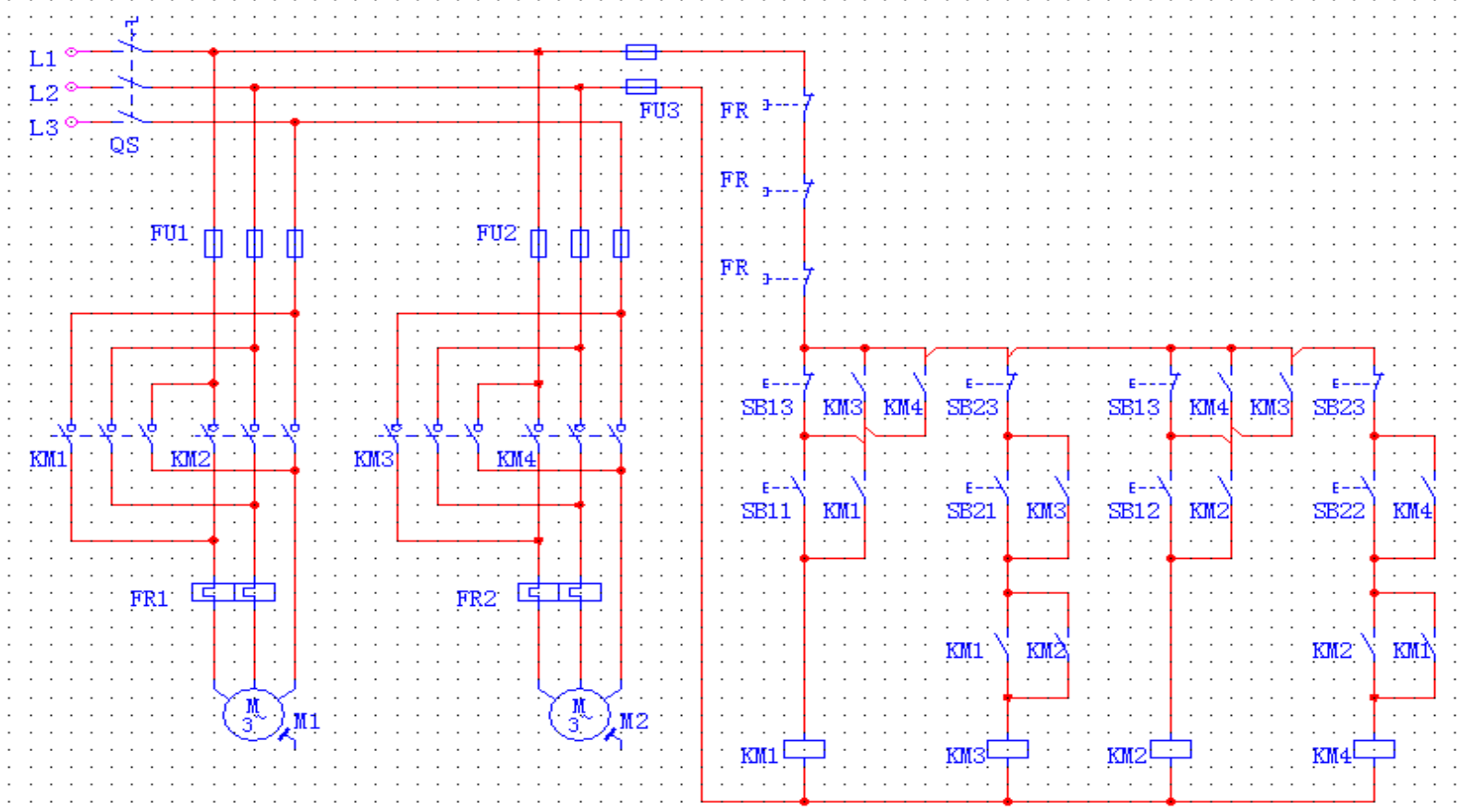

Figure 2. Belt control circuit diagram

After the conveyor remote speed control process, the process can start the motor circuit breaker closed manually, by the control center operator control interface PC click the Start button in the implementation. The PC startup command is a serial port to send the R232 or R485 to PLC, once the PLC receives startup command, you can output a high-level digital signals, to prompt contactor solenoid is energized. After this drive is powered by the serial port can receive $\mathrm{R} 232$ transmission to the start command to control the motor power, the motor drive in accordance with the internal preset start time to complete the soft start. When the conveyor system gradually reaches the rated speed, this operator can change the control parameters to achieve speed control through the PC control interface in real time.

\section{SYSTEM SOFTWARE STRUCTURE}

Before Industrial control system control mode is mainly to embody and completed by software program. This section focuses on the software programming of PLC control system and human-machine interface of configuration design, making the belt set control system on the basis of the hardware configuration is complete to achieve the required control function. System Control mode is local / remote control with switchable, mainly remote monitoring applications.

- Local control: without the aid of PC applications, directly by the operator through the PLC control and variable speed belt conveyor start-up, to realize the normal control system, but not for realtime monitoring the running state of belt conveyor;

- Remote control: with manual control and automatic control modes on the PC screen. The automatic control of PLC according to the signal from the sensor output frequency of self- control material transport speed; Manual control is a response to an operator according to what you see, start, stop, and reversing and speed control command, so as to realize the control of belt conveyor.

\section{A. King view communication with Siemens S7-200}

King view software as an excellent PC monitoring software, in many areas are widely used in remote monitoring[8]. King view regards every communication device as an external device, building a large number of device drivers as the communication interface to external devices to realize communication and external equipment. During the development process, according to equipment configuration wizard, step by step to complete the connection, configuration king and corresponding external device drivers can be realized.

King view driver using ActiveX technology, every driver is a COM object, this way make drivers and king view constitute a complete system, thus ensuring the efficient operation of the system[9]. Between the king view and PLC in this system uses the PPI (Point - to Point) way of communication. This mode is designed for 
Siemens S7-200 series developed a communication protocol, a master / slave protocol, PC machine-based station, S7-200 as slave.

\section{B. King view interface design process}

First, create a new project, make COM1 serial port settings, and then define the configuration king data variables.

Variables can be divided into two categories, basic type and special type. Basic type of variables is divided into two kinds of memory and I/O variables. The data collected from the lower machine, sent to the next bit machine instructions, such as belt conveyor speed, the power switch variables, all needs to be set to "I/O variables". Those who only use the variable within the king view, such as the calculation process of intermediate variable, can be set to "memory variables".

The basic types of variables can also be in accordance with the data type is divided into discrete, real, integer and string. After set the variable, then start to make graphic image, and graphics animation connection.

\section{MONITORING SYSTEM DEBUGGING AND DEMONSTRATION}

The monitoring system is a modular, researchers should adopt the method of single step debugging[10]. That validate the working process and the logical relationship from the stand is correct. First, build communication and choose communication mode in advance. After selecting the desired item, click "Test" options for network connectivity tests, including network transfer rate, the highest station address, the maximum and minimum delay time, setup time and so on.

After all parameters meet the practical requirements, click on the communication icon then working screen appears, to establish a network of communication between the personal computer and the device. After the communication is established, researchers will download the program from the computer to the PLC, and then start debugging.

Before the debugging process, researchers should remember that researchers must transform the PLC from the stop mode to run mode. In the upper machine to the next bit machine communication, ensure the correctness of the PLC control cabinet wiring at first, and then debug. After accurately select the remote address, download the program to the PLC, the following can be carried out automatically on the system hardware debugging upcoming LS1 into automatic mode, PLC in run mode , then click the " view of the state ". . In the state diagram of new numerical can fill in the required value of the variable, and controlled.

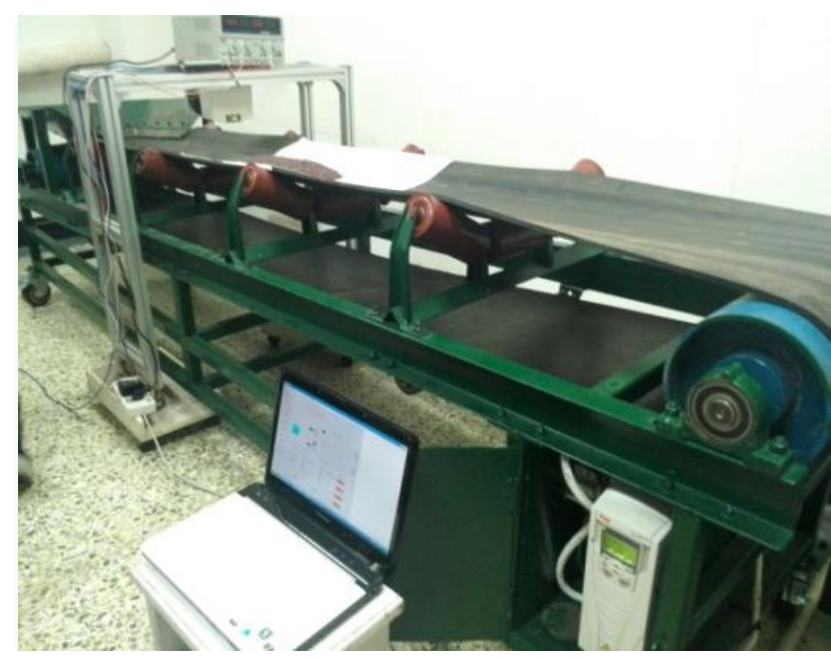

Figure 3. Part of test-bed experiment

Figure. 3 shows the testing stand part of the experiment. The trial showed that PC connects the lower machine by a communication protocol, and then controls belt conveyor. When researchers enter each parameter data on the host computer interface, then clip "start", it will drive belt to the desired speed positive \&negative, and collect the required data in real time.

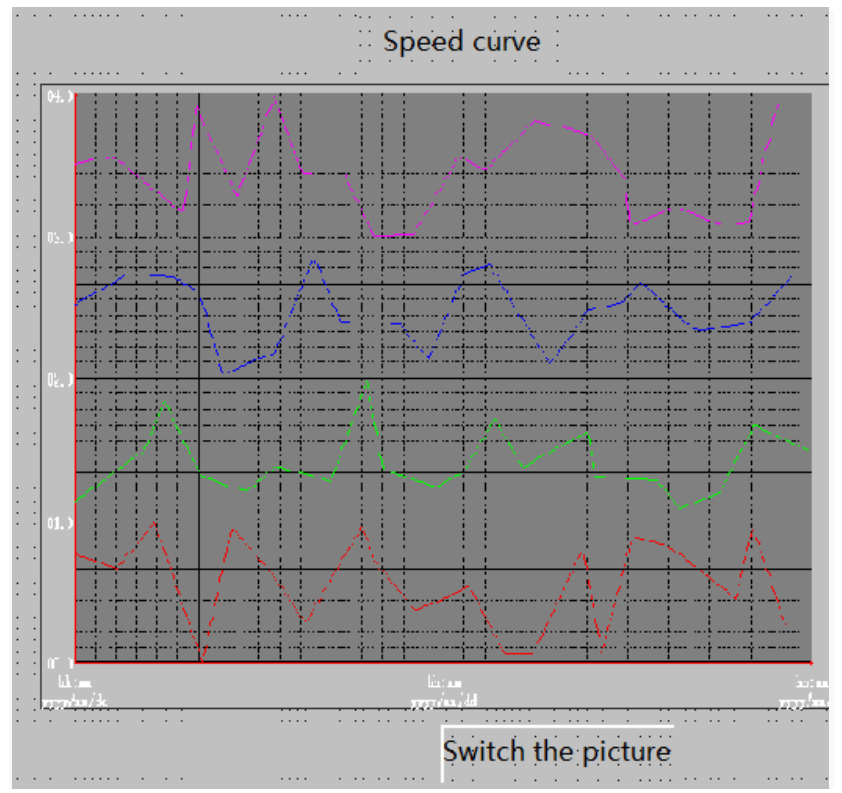

Figure 4. Speed curve

Figure. 4 is the certain speed curve in the process of the experiment shows. In this system, belt conveyor drive shaft connected to the speed sensor and application of the pulse sensor and converter module connected to through computer programming, to achieve the purpose of collecting the real-time speed of belt conveyor.

In this system, GSG8 mine speed sensor is used to detecting the speed, installed under the belt conveyer belt, for wire rope to tighten the speed wheel contact with the tape. When under the influence of the speed sensor in the encoder to produce light and light signal, the signal after amplification can produce high and low level pulse, then 
spread to the PLC, the processed generates the output signal.

\section{CONCLUSION}

Based on system design method described above, the author designed a monitoring system containing a PC interface, a lower control monitoring system and its supporting software required monitoring system.

This system has realized the function that king view software control belt conveyor on-site and remote through the PLC control cabinet, and can be realized using the PC interface to collect data, generate reports and other features. Actual running results show that the monitoring system is simple and convenient operation, operating normally, PC terminal cooperate well with PLC and frequency converter, transmission of data acquisition and control commands are accurate, and the whole control system is stable and reliable performance.

\section{ACKNOWLEDGMENT}

This work was supported by the Ministry of transport a nd applied basic research project of China (key platform gr ant No. 2013329811340), the Natural Science Fundation of the Jiangsu Higher Education Institution of China (Grant No. 14KJB5800 08), the Nature Science Foundation of Na ntong (Grant No. BK2014017).

\section{REFERENCES}

[1] The ministry of transport of the People's Republic of China China's shipping development report 2011 [M]. Beijing: people's traffic press, 2012:24 to 29.

[2] Zhang Tongxu, Xie Wenning. Port energy-saving reform of the belt conveyor technical measures $[\mathrm{J}]$. Journal of Marine science research, 2008, (1) : 34-38.

[3] Tan Chao. Dong Xiaojun. Based on virtual reality technology of remote monitoring system of underground belt conveyor. Coal mining machinery, 2009.30 (11).

[4] M. A. Alspaugh. Latest developments in belt conveyor technology [C], MIN-Expo 2004, Las Vegas, NV, USA, 27-30 Sept.,2004.

[5] Zhang S, Tang Y. Optimal scheduling of belt conveyor systems for energy efficiency-With application in a coal-fired power plant[C].Control and Decision Conference (CCDC), 2011 Chinese. IEEE, 2011: 1434-1439.

[6] Zheng Fengyi. The illustration of Siemens S7-200 series PLC application in 88 [M]. Electronic industry press, 2009.

[7] Wang Yu. PLC control and configuration design [M]. Electronic industry press, 2010.

[8] Su Yun, Pan Feng. Remote control system based on king view and PLC [J]. Industrial instrumentation and automation devices, 2004 (2) : 53-55.

[9] Wang Shanbin. Configuration software application guide Kingview Kingview and Siemens WinCC. [M]. Chemical industry press, 2011.

[10] G. M. Wang, X. J. Luo. Design and debugging of communication between controller and King view based on CAN bus. World Journal of Engineering, 2013, Vol.10 (4), 395-400. 